Revue musicale OICRM

\title{
Perception des intervalles harmoniques et apprentissage perceptuel
}

\section{Guillaume Fournier}

Volume 4, numéro 1, 2017

Apprendre et enseigner la musique au XXI ${ }^{\mathrm{e}}$ siècle. Nouvelles propositions pédagogiques

URI : https://id.erudit.org/iderudit/1040298ar

DOI : https://doi.org/10.7202/1040298ar

Aller au sommaire du numéro

\section{Éditeur(s)}

Observatoire interdisciplinaire de création et recherche en musique (OICRM)

\section{ISSN}

2368-7061 (numérique)

Découvrir la revue

Citer cet article

Fournier, G. (2017). Perception des intervalles harmoniques et apprentissage perceptuel. Revue musicale OICRM, 4(1), 17-43.

https://doi.org/10.7202/1040298ar
Résumé de l'article

Cet article élabore une nouvelle approche pédagogique pour étudier les intervalles harmoniques aux niveaux collégial et universitaire. Sont d'abord passés en revue les principaux mécanismes responsables de la perception des intervalles harmoniques, notamment la perception des hauteurs sonores, la capacité à discriminer les notes jouées simultanément, la perception des battements et l'harmonicité. En s'appuyant sur les mécanismes d'apprentissage perceptuel de Goldstone et Byrge (2015), différentes stratégies sont ensuite discutées pour isoler, stimuler et renforcer la perception des principaux attributs sonores nécessaire à la reconnaissance auditive des intervalles harmoniques. Une démarche d'enseignement intégrée est finalement proposée pour la salle de classe. 


\title{
Perception des intervalles harmoniques et apprentissage perceptuel
}

\author{
Guillaume Fournier
}

\begin{abstract}
Résumé
Cet article élabore une nouvelle approche pédagogique pour étudier les intervalles harmoniques aux niveaux collégial et universitaire. Sont d'abord passés en revue les principaux mécanismes responsables de la perception des intervalles harmoniques, notamment la perception des hauteurs sonores, la capacité à discriminer les notes jouées simultanément, la perception des battements et l'harmonicité. En s'appuyant sur les mécanismes d'apprentissage perceptuel de Goldstone et Byrge (2015), différentes stratégies sont ensuite discutées pour isoler, stimuler et renforcer la perception des principaux attributs sonores nécessaire à la reconnaissance auditive des intervalles harmoniques. Une démarche d'enseignement intégrée est finalement proposée pour la salle de classe.
\end{abstract}

Mots clés : apprentissage perceptuel ; éducation musicale ; formation auditive ; intervalles harmoniques; pédagogie musicale.

\begin{abstract}
This paper develops a new pedagogical approach to the study of harmonic intervals at the college and university levels. Mechanisms responsible for the perception of harmonic intervals, in particular the perception of tone, the ability to segregate notes played simultaneously, perception of beating and harmonicity, are first reviewed. Based on the perceptual learning mechanisms of Goldstone and Byrge (2015), different strategies to isolate, stimulate and reinforce the perception of the main sound attributes necessary for the auditory recognition of harmonic intervals are discussed. Lastly, an integrated approach to the teaching of harmonic intervals is suggested for the classroom.
\end{abstract}

Keywords: ear training; harmonic intervals; music education; music pedagogy; perceptual learning. 
Le parcours de formation des étudiants en musique de niveau collégial et universitaire comprend diverses activités de reconnaissance auditive destinées à développer leurs capacités perceptives et à améliorer leur compréhension du langage musical. Traditionnellement, les intervalles harmoniques figurent parmi les premiers éléments musicaux à être abordés, parce qu'ils constituent des notions théoriques élémentaires et qu'ils préparent à l'étude de l'harmonie (Balzano et Liesh 1982 ; Loh 2007 ; Ponsati et al. 2016 ; Samplaski 2005).

Pour faciliter leur apprentissage, plusieurs pistes ont été explorées : intégrer un nouvel intervalle par semaine (Killam, Lorton et Schubert 1975 ; Samplaski 2005) ; séparer les deux notes avant de les analyser (Ponsati et al. 2016 ; Wuthrich et Tunks 1989 ; Zatorre 1983) ; employer des chansons connues (Ponsati et al. 2016 ; Smith et al. 1994 ; Willems et Chapuis 1996) ; écouter le degré de consonance (Plomp et Levelt 1965 ; Schellenberg et Trainor 1996) ; attribuer aux intervalles des connotations psychologiques (Costa, Bitti et Bonfiglioli 2000 ; Maher 1976, 1980 ; Oelmann et Laeng 2009). En dépit de cette diversité d'approches à la disposition des étudiants, l'étude des intervalles harmoniques demeure une source de difficultés importantes pour un grand nombre d'entre eux (Balzano et Liesch 1982 ; Killam et al. 1975 ; Ponsati et al. 2016 ; Samplaski 2005 ; Wuthrich et Tunks 1989). Se pourrait-il que ces approches sous-estiment l'ampleur des défis perceptuels posés par l'étude des intervalles harmoniques?

$\mathrm{Au}$ cours de la dernière décennie, des avancées scientifiques importantes ont eu lieu en neuropsychologie, en cognition musicale et psychoacoustique. Elles ont permis de mettre en lumière les principaux mécanismes responsables de la perception musicale et de leur développement. Si l'importance des premières années de vie dans ce développement n'est plus à démontrer, il apparaît de plus en plus clairement que les systèmes perceptuels sont également dotés d'une plasticité leur permettant de poursuivre leur développement tout au long de la vie. Pour expliquer ce phénomène d'apprentissage perceptuel, Goldstone et Byrge (2015) ont récemment publié un modèle théorique qui nous apparaît pouvoir jeter un éclairage nouveau sur l'étude des intervalles harmoniques.

Dans cet article, nous tenterons de déterminer comment les mécanismes d'apprentissage perceptuel décrits par ces auteurs peuvent nous aider à concevoir des exercices adaptés pour renforcer la perception des principaux indices sonores contribuant à la reconnaissance auditive des intervalles harmoniques. Pour ce faire, nous présenterons d'abord leur modèle d'apprentissage perceptuel en mettant en évidence sa pertinence pour l'enseignement des intervalles harmoniques. Nous exposerons ensuite les principaux mécanismes perceptuels sollicités durant l'écoute des intervalles harmoniques, notamment ceux qui sont responsables de la perception des notes isolées (Oxenham 2013 ; Stainby et Cross 2009), de la friction (Lee 2011 ; Thompson 2013) et de l'harmonicité (Micheyl et Oxenham 2010 ; Thompson 2013). Enfin, en nous appuyant sur ces connaissances scientifiques, nous proposerons une nouvelle démarche d'enseignement des intervalles harmoniques qui, en amont des approches traditionnelles, visera à stimuler chez l'étudiant un véritable apprentissage perceptuel. 
APPRENTISSAGE PERCEPTUEL

Les capacités perceptuelles sont-elles fixes - déterminées par une maturation biologique - ou peuvent-elles se développer lorsqu'on les soumet à un entraînement approprié ? À cette question, Goldstone et Byrge offrent une réponse sans équivoque : "perceptions can be learned ". Pour en arriver à cette conclusion, ces deux auteurs se sont appuyés sur de nombreuses recherches empiriques réalisées après d'enfants et d'adultes. Il apparaît clairement que les stimuli présents dans l'environnement de l'enfant influencent son développement perceptuel (Quinn et Bhatt 2006 ; Needham, Dueker et Lockhead 2005). À l'âge adulte, plusieurs éléments nous indiquent que les systèmes perceptuels sont dotés d'une plasticité leur permettant de continuer à s'optimiser afin de mieux percevoir les stimuli nécessaires à la réalisation des tâches. On observe fréquemment cette plasticité chez certains experts dont le travail répétitif autour d'une même activité stimule le développement d'habiletés perceptuelles très spécialisées. Chez les ornithologues, par exemple, la région du cortex visuel responsable de la détection des mouvements se développe davantage que chez la population en général, ce qui leur permet de repérer plus facilement les petits déplacements rapides dans de nouvelles circonstances (Tanaka et Curran 2001). Un second exemple est celui des radiologistes, qui réussissent à percevoir sur une image des contrastes d'une intensité nettement plus faible que chez la plupart des gens (Sowden, Davies et Roling 2000). Enfin, plusieurs recherches expérimentales ont confirmé qu'il était possible d'améliorer les perceptions sensorielles à 1'âge adulte par le biais d'un l'entraînement (Bao et al. 2010 ; Furmanski, Schluppeck et Engel 2004 ; Weinberger 1993).

\section{Mécanismes d'apprentissage perceptuel}

De façon générale, le modèle de Goldstone et Byrge repose sur l'idée que les systèmes perceptuels s'adaptent continuellement à leur environnement pour réagir plus efficacement aux stimuli qui se révèlent importants pour le fonctionnement de l'individu. Les perceptions se développent donc au fil des expériences et tout est susceptible d'y contribuer : par exemple, la langue maternelle, la nature des tâches à accomplir, les connaissances conceptuelles. De manière plus précise, les auteurs exposent trois mécanismes permettant d'expliquer comment se réalisent ces apprentissages perceptuels.

\section{Attention}

Le premier mécanisme est celui de l'attention. Bien qu'on ait trouvé quelques exemples où l'exposition passive à un stimulus sensoriel a provoqué des changements perceptuels (Watanabe, Nanez et Sasaki 2011), c'est essentiellement en orientant activement son attention vers un attribut sensoriel considéré comme important qu'on pourra entraîner un apprentissage perceptuel. Toutefois, nous le verrons en détail plus loin, la capacité d'orienter son attention vers certains attributs sensoriels est souvent limitée par la présence des catégories perceptuelles existantes chez l'individu. Par exemple, il est très difficile de percevoir les phonèmes d'une nouvelle langue 
lorsque le système auditif est spécialisé pour filtrer les sonorités de sa langue maternelle (Goldstone et Hendrickson 2010).

\section{Fusion}

Cette perception catégorielle est la conséquence du second mécanisme d'apprentissage perceptuel : la fusion. Pour des raisons d'efficacité, les systèmes perceptuels cherchent à regrouper les différents attributs sensoriels en unités plus simples et globales. Par exemple, la couleur contient de nombreux attributs sensoriels perceptibles, dont la luminosité et la saturation. Comme ils se présentent naturellement en cooccurrence et qu'il n'est généralement d'aucune utilité de savoir les distinguer, la plupart des gens les regroupent naturellement et, en conséquence, ne parviennent plus à les percevoir distinctement (Garner 1976). La fusion des attributs sensoriels est un mécanisme essentiel qui permet à l'individu de continuer à bien percevoir son environnement en présence de variations.

\section{Discrétisation}

Pour créer de nouvelles catégories perceptuelles, le modèle prévoit un troisième mécanisme, qui fait exactement le contraire de la fusion : la discrétisation. Plusieurs recherches ont montré qu'il est possible d'apprendre à distinguer des attributs qui sont fusionnés. Une manière efficace d'y parvenir consiste à réaliser en alternance deux tâches qui nécessitent la perception des attributs précis que l'on souhaite différencier. En employant cette méthode à la perception visuelle, Goldstone et Steyvers (2001) sont parvenus à former de nouvelles catégories perceptuelles.

En bref, l'être humain s'est doté de systèmes perceptuels qui s'adaptent constamment pour percevoir plus efficacement les stimuli présents dans son environnement. Lorsqu'une perception globale est requise, les attributs perceptuels fusionnent au point de les rendre indiscernables. Lorsque, au contraire, la perception doit être plus précise, une phase d'apprentissage perceptuel est amorcée et conduit à la discrétisation des stimuli. Cette vision adaptative de la perception nous apparaît pleinement appropriée pour aborder différemment l'étude des intervalles harmoniques. En effet, si une part des difficultés rencontrées par les étudiants est d'origine perceptuelle, trouver des moyens concrets pour stimuler la perception des attributs sonores pertinents à l'étude des intervalles harmoniques nous apparaît être une approche très prometteuse.

La démarche d'apprentissage que proposent Goldstone et Byrge est en principe assez simple d'application pour l'étude des intervalles harmoniques : il nous faut trouver des moyens pour aider l'étudiant à prendre conscience des différents attributs sonores qui composent les intervalles harmoniques, puis créer des tâches pertinentes qui stimuleront leur discrétisation. Quels attributs sonores contribuent à la reconnaissance des intervalles harmoniques?

\section{PeRception DES INTERVALLES HARMONiQues}

L'examen des principales approches employées pour la reconnaissance des intervalles harmoniques peut nous aider à identifier les attributs sonores à retenir. 
Deux approches sont fréquemment préconisées par les pédagogues. La première consiste à extraire les deux notes qui composent l'intervalle harmonique pour ensuite les analyser comme s'il s'agissait d'intervalles mélodiques (Ponsati et al. 2016 ; Rogers 2004 ; Wuthrich et Tunks 1989 ; Zatorre 1983). Sur le plan perceptuel, cette démarche requiert la capacité de percevoir distinctement deux notes jouées simultanément.

La seconde approche pédagogique consiste à écouter "globalement " l'intervalle harmonique, notamment en se référant à son degré de consonance (Karpinski 2000 ; Rogers 2004). Bien que cette approche puisse sembler nettement plus subjective, nous verrons qu'elle repose sur des mécanismes perceptuels bien documentés dans la littérature.

\section{Percevoir distinctement les notes entendues simultanément}

La capacité à séparer différentes notes entendues simultanément représente une tâche particulièrement difficile, tant pour la perception des intervalles harmoniques que des accords (Demany et Ramos 2005 ; Huron 1989 ; Ponsati et al. 2016 ; Roederer 2008). Lorsque la tâche se déroule dans un contexte musical, les auditeurs peuvent exploiter différents indices tels le timbre instrumental, la conduite mélodique des voix, la provenance physique des sons et les légères différences de synchronisation (Deutch 2013 ; Goldstein 2010 ; Stainby et Cross 2009). Mais, lorsque les notes sont produites simultanément sans contexte musical, la tâche devient très difficile pour l'oreille. Commençons par expliquer comment l'oreille y parvient avec des sons simples, sans harmoniques.

\section{Perception des sons simples}

On peut distinguer deux mécanismes permettant ce type de discrimination (Roederer 2008). Le premier se situe au niveau mécanique et est lié aux propriétés physiques de la membrane basilaire. Grâce aux travaux de Georg von Békésy, nous savons que cette membrane, située au cœur de la cochlée, joue un rôle essentiel dans la perception des hauteurs. Lorsqu'elle est traversée par une onde sonore, elle réagit en vibrant par sympathie à un endroit très précis où se logent les cellules ciliées internes qui, elles, répondent chacune à une bande de fréquences très étroite. Ces cellules convertissent l'énergie mécanique en influx nerveux et les acheminent aux aires auditives afin d'être interprétées (Goldstein 2010). Or, lorsque deux notes rapprochées sont entendues simultanément, les fréquences mettent en vibration la même région de la membrane basilaire, ce qui engendre certaines conséquences. D'abord, une seule note peut être encodée dans le nerf auditif, ce qui constitue en soi une limite mécanique à la discrimination des fréquences. Elle s'accompagne ensuite d'une fluctuation d'intensité ressemblant à un battement (Plomp et Levelt 1965 ; Roederer 2008). Enfin, la perception de deux notes simultanément produit un effet de masquage, qui a pour effet de relever le seuil à partir duquel un son devient audible (Goldstein 2010); cet effet est d'autant plus important lorsque les notes sont rapprochées. L'intensité sonore des notes devient alors un paramètre déterminant dans la capacité à discriminer les fréquences. Ainsi, les notes rapprochées sont plus difficiles à discriminer en raison de 
la présence de battements et du phénomène de masquage. L'exemple audio 1 permet d'illustrer ces premiers mécanismes perceptuels.

L'exemple audio 1 émettra dans chaque oreillette une note légèrement différente $(220 \mathrm{~Hz}$ dans l'oreillette droite ; $223 \mathrm{~Hz}$ dans l'oreillette gauche). À l'aide d'une paire d'écouteurs intra-auriculaires, écoutez l'extrait audio dans les trois conditions qui suivent. D'abord, en employant une seule oreillette à la fois, vous devriez percevoir deux sons purs totalement dépourvus de battement ou d'ondulation. Ensuite, en réunissant les deux oreillettes de façon à pouvoir les écouter simultanément avec une même oreille, vous devriez percevoir un battement régulier de forte intensité. Enfin, en utilisant les deux oreillettes simultanément de façon normale, vous devriez percevoir une très légère sensation de profondeur, d'ondulation et de mouvement, sans toutefois percevoir le battement accentué de la seconde condition d'écoute. Les battements tirent essentiellement leur origine dans la cochlée.

Exemple audio $1:$ Perception des battements. Écouter.

La discrimination des fréquences ne se limite pas qu'au travail mécanique de l'oreille. La manière de traduire l'énergie mécanique en influx nerveux tout comme les moyens employés par le cortex auditif pour traiter l'information contribuent également à la réalisation de cette tâche complexe. Au niveau de la cochlée, le codage des hauteurs sonores se déroule de deux façons. D'abord, chaque cellule ciliée interne, qui réagit, rappelons-le, à une bande très étroite de fréquences, vient stimuler une région spécifique du cortex auditif. On parle ici de codage spatial (Chouard 2001 ; Goldstein 2010 ; Oxenham 2013). Avec des techniques d'imagerie cérébrale, il est d'ailleurs possible de visualiser l'emplacement précis de chacune des notes entendues dans le cerveau (Bendor et Wang 2005 ; Goldstein 2010). Ensuite, chaque neurone tente de synchroniser le rythme de ses impulsions électriques avec la fréquence entendue. On parle ici du codage temporel. Le nerf auditif ayant besoin d'une période de repos entre chaque impulsion électrique, ce codage s'effectue à une fréquence maximale de $1000 \mathrm{~Hz}$, ce qui limite la perception fine des fréquences à partir de ce seuil (do6). En effet, les cellules ciliées doivent ralentir la cadence de leurs impulsions électriques afin de pouvoir se recharger. Par exemple, pour encoder la note si6 $(1975 \mathrm{~Hz})$, les cellules ciliées se synchronisent avec seulement la moitié des ondulations sonores ${ }^{1}$. Au-delà d'environ $4000 \mathrm{~Hz}$, seul le codage spatial peut contribuer à la sensation de hauteur sonore (voir les exemples audio 2 et 3 ). En somme, ces deux modes d'encodage contribuent de manière différente à la perception des hauteurs sonores. La perte d'efficacité du second mécanisme dans les fréquences plus aigües explique une partie des difficultés à discriminer deux notes entendues simultanément.

1 Selon Chouard (2001), ce phénomène pourrait être à l'origine de la similitude perceptuelle des octaves. 
Pour observer la perte de discrimination des fréquences qui survient au-delà du seuil des $4000 \mathrm{~Hz}$, l'exemple audio 2 présente deux courtes séquences mélodiques de part et d'autre de cette limite. Voyez comment le jugement de hauteur est nettement plus difficile au-delà de $4000 \mathrm{~Hz}$.

Dans l'exemple audio 3, cinq intervalles de secondes majeures sont produits dans les régions de $500 \mathrm{~Hz}$, $1000 \mathrm{~Hz}, 2000 \mathrm{~Hz}, 4000 \mathrm{~Hz}$ et $8000 \mathrm{~Hz}$. Constatez à quel point il devient graduellement plus difficile de percevoir distinctement les deux notes au-delà de $1000 \mathrm{~Hz}$.

Exemples audio 2 et 3 : Codage temporel au-delà de $1000 \mathrm{~Hz}$. Écouter l'exemple 2 et l'exemple 3.

\section{Perception des sons complexes}

Lorsque les sons entendus sont joués par de vrais instruments, un autre mécanisme perceptuel participe à la perception des hauteurs. Il s'agit de la capacité à résoudre la structure des harmoniques associés à une fréquence fondamentale. En effet, les propriétés acoustiques de la voix humaine et des instruments de musique font en sorte que chaque son musical est accompagné de fréquences secondaires, appelées harmoniques, respectant la distribution présentée dans la figure 1.

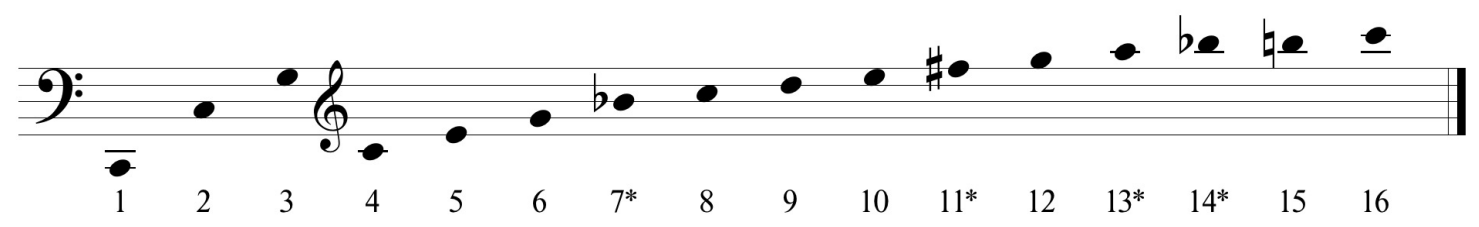

Figure 1 : Série des harmoniques naturels.

Plusieurs expériences ont montré que la présence de ces harmoniques dans le son musical renforce la perception de la fréquence fondamentale (Bernstein et Oxenham 2008 ; Goldstein 2010 ; Oxenham 2013 ; Roederer 2008 ; Stainsby et Cross 2009). Cette sensation des hauteurs découlant de la résolution des harmoniques contribue à contrecarrer les effets de masquage (Oxenham 2013) et aide à discriminer les différentes sources sonores (Stainsby et Cross 2009). Sur le plan biologique, l'existence de ce mécanisme permettrait également de prévenir la détérioration de l'audition due à la destruction de cellules ciliées internes (Goldstein 2010). Leur rôle est si grand dans la perception des hauteurs que l'écoute des harmoniques est suffisante en elle-même pour percevoir la fréquence fondamentale, comme l'illustre l'exemple audio 4.

Afin d'illustrer le rôle des harmoniques dans la perception de la fréquence fondamentale, nous avons manipulé l'échantillon sonore d'une contrebasse jouant la note do2 $(64 \mathrm{~Hz})$ de façon à retirer d'abord la fréquence fondamentale, puis graduellement toutes les fréquences inférieures à $1500 \mathrm{~Hz}$. Au fil des transformations, observez comment la perception de la fréquence fondamentale perdure pendant l'élimination des 21 premiers harmoniques.

Exemple audio 4 : Résolution des harmoniques. Écouter.

Le survol des mécanismes responsables de la discrimination des fréquences nous aide à mieux comprendre la nature des difficultés rencontrées lorsqu'on souhaite 
percevoir distinctement les notes qui composent les intervalles harmoniques. Nous saisissons mieux pourquoi les notes très rapprochées sont plus difficiles à percevoir, pourquoi nous perdons de la définition au-delà de $1000 \mathrm{~Hz}$ et comment l'oreille peut tirer profit de la présence des harmoniques pour percevoir la fréquence fondamentale dans les sons complexes.

\section{Perception de la consonance}

La seconde approche fréquemment employée pour étudier les intervalles harmoniques consiste à écouter leur " couleur " globale, notamment en prêtant attention à leur degré de consonance (Karpinski 2000 ; Rogers 2004). Bien que l'appréciation de la consonance soit fortement influencée par des facteurs culturels, plusieurs chercheurs ont tenté d'isoler les mécanismes perceptuels qui en sont responsables (voir, par exemple, Thompson 2013). La plupart des recherches intéressées à la consonance ont porté sur deux paramètres perceptuels : la friction et 1'harmonicité (Lee 2011 ; Bernstein et Oxenham 2008).

\section{Friction}

Plusieurs musiciens et chercheurs ont suggéré que la présence des battements pouvait être responsable du degré de consonance perçu des intervalles harmoniques (Lee 2011 ; Thompson 2013). Les musiciens avaient en effet remarqué que la présence de battements faisait diminuer la sensation de la justesse et qu'elle augmentait la sensation de dissonance, comme en témoignent de nombreux textes publiés sur l'harmonie et l'accordage des instruments durant le $\mathrm{XVII}^{\mathrm{e}}$ siècle (Keislar 1991 ; Lee 2011). Par exemple, en 1877, Helmholtz observait que le nombre d'harmoniques communs entre deux sons musicaux permettait de prédire assez précisément le degré de consonance perçu des intervalles (Stolzenburg 2015). Mais, c'est en 1965 que l'on parvint à situer plus précisément le mécanisme responsable de la perception de la friction : la résolution des harmoniques dans la cochlée. En effet, les fréquences rapprochées sur la membrane basilaire ne peuvent être perçues distinctement et induisent des battements qui contribuent à augmenter la sensation de dissonance. Or, lorsque deux sons musicaux sont entendus simultanément, leurs harmoniques peuvent également interagir dans la cochlée. Selon le modèle de Plomp et Levelt (1965), plus les sons contiennent d'harmoniques pouvant être résolus (communs ou suffisamment distancés entre eux), plus la sensation de consonance sera grande. À l'inverse, plus les harmoniques sont rapprochés, moins ils pourront être résolus dans la cochlée et plus les intervalles seront accompagnés de battements augmentant la sensation de rudesse (Bidelman et Krishman 2009). Plusieurs recherches se sont intéressées à cette explication de la consonance et ont confirmé sa robustesse. Par exemple, Kameoka et Kuriyagawa (1969, cités par McLachlan et al. 2013) ont observé que l'intensité des harmoniques des différents timbres instrumentaux affectait la perception de la consonance. De son côté, Keislar (1991) a montré que l'on pouvait altérer la perception de la consonance en manipulant artificiellement la hauteur de certaines harmoniques. Ainsi, pour l'étude des intervalles harmoniques, les interactions entre les harmoniques constituent des indices essentiels à percevoir. 
L'usage des battements pour l'identification des harmoniques comporte toutefois quelques limites. Comme nous l'avons expliqué précédemment, la sensation des battements varie en fonction de l'intensité des harmoniques des sons entendus (Kameoka et Kuriyagawa 1969). Ceci signifie que certains intervalles sembleront plus ou moins dissonants selon les instruments utilisés ou encore selon la manière de les jouer. De plus, la cochlée résout beaucoup plus difficilement les fréquences se situant dans les registres extrêmes (Oxenham 2013). Dans le grave, les harmoniques sont beaucoup plus difficiles à résoudre et entraînent une perception de rudesse plus grande pour tous les intervalles.

Malgré la pertinence du modèle des battements, quelques phénomènes résistent à ses explications (Bowling et Purves 2015 ; McDermott et Oxenham 2008). Par exemple, en manipulant en laboratoire les stimuli sonores afin de réduire artificiellement le nombre de battements perçus (voir exemple audio 5), on a remarqué que la sensation de dissonance ne se dissipait jamais complètement. De plus, un cas d'amusie documentée par Cousineau et al. (2012) suggère que le jugement sur la consonance ne repose pas uniquement sur la perception des battements. Une seconde explication gagne en popularité : celle de 1'harmonicité (Langner et Ochse 2006 ; McDermott, Lehr et Oxenham 2010).

L'exemple audio 5 répartit entre les deux oreilles les deux notes d'une seconde mineure, ce qui élimine entièrement les frictions dans la cochlée. Pourtant, une sensation de dissonance subsiste.

Exemple audio 5 : Perception de la dissonance malgré l'absence de battements. Écouter.

\section{Harmonicité}

Les musiciens ont remarqué depuis fort longtemps qu'il semblait exister des liens entre la sensation de consonance et la série des harmoniques naturels. Comme nous le rappellent Bowling et Purves (2015), Jean-Philippe Rameau, au XVIII ${ }^{\mathrm{e}}$ siècle, croyait que la série harmonique était à l'origine de l'harmonie tonale. Trois explications de la consonance admettent aujourd'hui un rôle de la série des harmoniques. La première, celle du schéma spectral ${ }^{2}$ (Deutch 2013 ; Oxenham 2013), suggère que l'enfant se familiariserait très tôt avec le schéma régulier des harmoniques, notamment grâce au contact de la voix humaine (Bowling et Purves 2015). Lorsque deux notes seraient entendues simultanément, les intervalles harmoniques qui correspondent le plus fortement à ce schéma seraient alors perçus comme familiers et, conséquemment, plus consonants.

La seconde explication est très similaire, mais fait intervenir un mécanisme de perception de la hauteur que nous avons décrit plus haut, à savoir la capacité à inférer la fréquence fondamentale à partir des harmoniques. Comme nous l'avons mentionné, la résolution des harmoniques contribue très fortement à la perception de la fréquence fondamentale. Or, lorsque deux notes musicales sont entendues simultanément, 
l'oreille tente de les résoudre de la même manière qu'elle le fait avec les harmoniques naturels, c'est-à-dire en recherchant une fréquence fondamentale commune aux deux sons (Micheyl et Oxenham 2010 ; Thompson 2013). L'exemple audio 6 illustre la manière dont l'harmonicité contribue à la perception de la consonance de l'intervalle de sixte mineure. Lorsque deux notes sont entendues simultanément, le système auditif tente de les situer dans un même
schéma spectral. Dans l'exemple audio 6, les notes mi4 et do5 qui forment l'intervalle de sixte mineure peuvent
appartenir à un même schéma spectral, celui de la fondamentale do2.

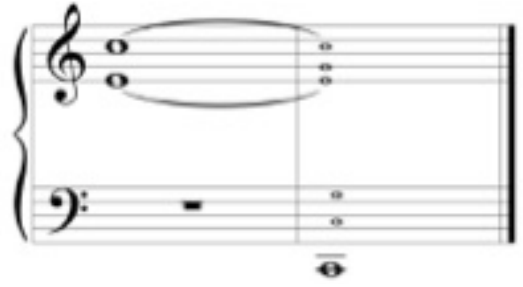

Exemple audio 6: Perception de l'harmonicité. Écouter.

Ainsi, lorsque deux notes entendues peuvent appartenir à une même fréquence fondamentale, l'oreille en perçoit virtuellement la présence (Lee 2011 ; Tramo et al. 2001). Cette note, nécessairement plus grave, est absente du stimulus acoustique. Non seulement est-elle perçue subjectivement, mais on peut également la détecter dans le cortex auditif (Lee 2011). Bien que l'on pourrait croire que ce phénomène soit d'origine acoustique, des recherches ont montré que la perception de cette fréquence était accentuée par l'entraînement musical (ibid. ; McDermott, Lehr et Oxenham 2010). Nous pourrions ajouter que cette explication est en cohérence avec le modèle d'apprentissage perceptuel de Goldstone et Byrge dans la mesure où la présence continuelle des schémas spectraux dans les sons musicaux pourrait expliquer la fusion perceptuelle de ces attributs sonores.

Des chercheurs en neuropsychologie ont proposé une troisième explication qui s'appuie sur le mécanisme de codage temporel des hauteurs sonores (Langner et Orshe 2006). Rappelons-nous que les impulsions électriques qui sont acheminées vers le cortex auditif se synchronisent avec les différentes fréquences qui sont entendues simultanément (codage temporel). En superposant ces différentes fréquences, des coïncidences apparaissent de manière périodique. Selon ces chercheurs, cette régularité serait à l'origine de la perception de la fréquence fondamentale virtuelle. Plus les impulsions sont synchronisées, plus les intervalles paraissent consonants (Bidelman et Krishman 2009 ; Lee 2011 ; Stolzenburg 2015 ; Thompson 2013). Ici également, l'entraînement musical amplifie la perception de l'harmonicité (Lee 2011).

\section{COMMENT AMÉLIORER LA PERCEPTION DES INTERVALLES HARMONIQUES?}

Dans la seconde partie de cet article, nous avons présenté les principaux mécanismes perceptuels qui sont à l'œuvre durant l'étude des intervalles harmoniques. Mais qu'en est-il pour leur développement à l'âge adulte ? Comment peut-on stimuler la perception de la fréquence fondamentale, des battements et de l'harmonicitéchez 
l'étudiant adulte ? Cette section examine diverses pistes pour conduire à la discrétisation des paramètres sonores qui nous intéressent. À l'issue de cette réflexion, nous proposerons une démarche d'enseignement des intervalles harmoniques qui combinera les pistes nous apparaissant être les plus pertinentes pour l'enseignement des intervalles harmoniques au niveau collégial ou universitaire.

Potentiel de développement

Comme le prévoit le modèle d'apprentissage perceptuel de Goldstone et Byrge, plusieurs recherches ont montré que l'expérience musicale améliorait la discrimination fine des fréquences (Goldstein 2010 ; Musacchia et al. 2007), la perception des harmoniques supérieurs (Lee et al. 2009) et l'harmonicité (McDermott, Lehr et Oxenham 2010). De plus, les zones dédiées à la représentation des hauteurs sonores sont beaucoup plus étendues chez les musiciens que chez les non-musiciens (Pantev et al. 1998 ; Schneider et al. 2002). Malgré les limites méthodologiques inhérentes à ce type de recherches réalisées auprès de musiciens professionnels, la plasticité cérébrale induite par la pratique musicale est désormais un fait établi (voir Trainor et Hannon 2013). De surcroît, plusieurs études expérimentales ont montré que les non-musiciens pouvaient réaliser des progrès significatifs sur le plan de la discrimination fine des fréquences grâce à l'entraînement (Bosnyak, Eaton et Roberts 2004 ; Carcagno et Plack 2011 ; Zarate, Ritson et Poeppel 2010). Mais, comment le modèle d'apprentissage perceptuel peut-il nous aider à concevoir des exercices adaptés à l'étude des intervalles harmoniques?

\section{Les clés de l'apprentissage perceptuel}

Comme nous l'avons vu lorsque nous présentions les mécanismes d'apprentissage perceptif de Goldstone et Byrge, la discrétisation, cette capacité à former de nouvelles catégories perceptuelles, survient lorsque les tâches à accomplir nécessitent la perception d'attributs sensoriels jusqu'ici fusionnés. Par exemple, les catégories perceptuelles développées pour percevoir les phonèmes de sa langue maternelle se révèlent rapidement inadaptées pour apprendre une nouvelle langue. Cette situation forcera l'individu à prêter attention à de nouvelles sonorités et à redéfinir les catégories perceptuelles existantes. Sur le plan pédagogique, la première clé de l'apprentissage perceptuel réside donc dans la mise en place d'un contexte favorisant la redéfinition des catégories perceptuelles. Autrement dit, la nature de la tâche à accomplir doit mettre en évidence pour l'étudiant la nécessité de prêter davantage attention à de nouveaux attributs sensoriels. Dans le cas de l'apprentissage d'une langue seconde, dès que l'on souhaite mettre en place une communication orale, il devient rapidement incontournable de percevoir et d'énoncer de nouveaux phonèmes.

La seconde clé de l'apprentissage perceptuel repose sur la sélection de moyens appropriés pour attirer l'attention autour des attributs sensoriels que l'on souhaite différencier. Dans les recherches expérimentales réalisées dans le domaine de la perception visuelle, les auteurs ont fréquemment eu recours à des stimuli conçus pour introduire une variation sur un paramètre très ciblé. Par exemple, en faisant 
varier séparément le niveau de saturation et de luminosité sur une série d'images, Goldstone a réussi à entraîner les participants à discriminer ces deux paramètres dans la perception de la couleur (Goldstone 1994). Dans le contexte de la salle de classe, nous croyons que les tâches à réaliser devraient non seulement créer un contexte favorisant la recomposition des catégories perceptuelles, mais qu'elles devraient aussi être conçues de façon à contraindre l'étudiant à prêter attention aux différents attributs sensoriels que l'on souhaite étudier. En clair, la tâche proposée à l'étudiant ne devrait pas pouvoir être résolue autrement qu'en apprenant à mieux percevoir les attributs sensoriels qui nous intéressent. Dans le cas des intervalles harmoniques, nos exercices devront donc amener l'étudiant à mieux percevoir la fréquence fondamentale, les interactions entre les harmoniques et l'harmonicité. Voyons comment y arriver.

\section{Perception fine de la fréquence fondamentale}

Pour renforcer la perception de la fréquence fondamentale, une première piste à retenir est celle de l'entraînement à la discrimination fine des fréquences. En effet, pour résoudre une tâche de discrimination fréquentielle, on doit nécessairement prêter attention à la fréquence fondamentale. Étant donné que la discrimination fine des fréquences est sollicitée quotidiennement chez les musiciens, notamment durant l'accordage des instruments de musique, apprendre à poser de meilleurs jugements de justesse nous apparaît être un objectif susceptible de mobiliser l'intérêt de l'étudiant. La pertinence de cette tâche paraît évidente pour les musiciens qui accordent eux-mêmes leur instrument de musique, mais ceux qui jouent d'un instrument dont l'accordage est habituellement confié à des spécialistes, comme le piano ou l'orgue, demeurent tout aussi concernés : ils doivent apprendre à poser d'excellents jugements de justesse afin notamment de mieux remplir leur rôle de " gardien " de la justesse auprès des autres musiciens.

Dans la plupart des recherches réalisées en laboratoire, les stimuli sonores employés pour l'entraînement à la discrimination fine des fréquences étaient des sons simples, sans harmoniques. Cette forme de stimulus présente de nombreux avantages dans un contexte de recherche, mais plusieurs raisons nous motivent à plutôt proposer l'utilisation de véritables timbres instrumentaux dans le contexte de la formation auditive. D'abord, sur le plan philosophique, l'usage de fréquences simplifiées nous apparaît hautement discutable dans la mesure où les habiletés acquises dans les cours de formation auditive devraient refléter le mieux possible les véritables contextes musicaux où ils seront employés. Ensuite, il est clair que les harmoniques jouent un rôle important dans la perception de la fréquence fondamentale. Un entraînement qui les évacuerait risquerait de dénaturer le processus naturel de perception des hauteurs sonores. Enfin, quelques recherches suggèrent que les possibilités de transfert seraient plus grandes lorsqu'on emploie une diversité de timbres instrumentaux pour étudier les intervalles harmoniques (Demany et Semal 2002 ; Miyazono, Glasberg et Moore 2010). Pour ces raisons, il nous apparaît donc essentiel d'aborder la discrimination fine des fréquences avec de véritables timbres instrumentaux.

Considérant ce que nous venons d'énoncer, le moyen que nous privilégions pour stimuler la discrimination fréquentielle est celui d'accorder des instruments de 
musique (ou des enregistrements de véritables timbres instrumentaux) en employant des notes de références jouées de manières successives. En les jouant mélodiquement plutôt qu'harmoniquement, l'étudiant devra obligatoirement prêter attention à la fréquence fondamentale, peu importe les variations induites par les timbres instrumentaux et les interactions entre les harmoniques. L'étudiant devrait être encouragé à poursuivre son entraînement au-delà des limites de son propre instrument. Étant donné que les perceptions sont influencées par les expériences individuelles, on peut s'attendre, par exemple, à ce que les flûtistes éprouvent davantage de difficulté à percevoir les fréquences graves que les contrebassistes. Par ailleurs, afin de tenir compte de la perception plus ardue dans les registres extrêmes, l'entraînement ne devrait pas négliger les notes graves ainsi que celles dépassant $4000 \mathrm{~Hz}$.

\section{Extraction des fréquences fondamentales}

Une seconde piste de développement est celle d'entraîner directement l'étudiant à reconnaître distinctement deux notes jouées simultanément. Nous avons vu plus haut que les difficultés d'extraction s'expliquent en grande partie par la présence de deux interactions possibles : les battements et l'harmonicité. Les battements étaient perçus lorsque les fréquences fondamentales ou les harmoniques étaient trop rapprochés pour être résolus distinctement dans la cochlée, alors que l'harmonicité survenait lorsque deux notes pouvaient appartenir à un même schéma spectral. Ces deux mécanismes expliquent pourquoi la perception distincte des notes est plus difficile avec les intervalles générant de nombreux battements, comme les secondes et le triton, ainsi qu'avec ceux présentant de fortes similitudes spectrales, comme l'unisson et l'octave.

Afin de stimuler la capacité de l'oreille à outrepasser ces limites perceptuelles, nous proposons d'abord de miser sur le phénomène de résolution des harmoniques. En effet, les harmoniques permettent de percevoir des fréquences fondamentales absentes ou qui seraient masquées par un nombre trop élevé de battements dans la cochlée. Pour tirer profit du mécanisme de résolution des harmoniques, il apparaît toutefois essentiel d'employer une diversité de timbres instrumentaux, puisque l'intensité des harmoniques diffère grandement d'un instrument de musique à l'autre. Ensuite, pour lutter contre la fusion perceptuelle liée à l'harmonicité, l'exercice d'extraction des fréquences devrait être réalisé avec un seul nouvel intervalle harmonique à la fois. Ceci permettra à l'étudiant de se familiariser avec les fusions spectrales propres à chacun des intervalles tout en renforçant sa capacité à extraire les fréquences fondamentales pour une diversité de timbres instrumentaux. Enfin, pour aider l'étudiant à accorder son attention aux bons indices perceptuels, nous proposons d'employer la démarche que Goldstone et Steyvers (2001) ont utilisée pour entraîner les participants à reconnaître les attributs visuels, à savoir, alterner deux stimuli qui se distinguent par l'attribut qui nous intéresse. Ainsi, pour aider l'étudiant à percevoir distinctement deux notes jouées simultanément, elles devraient d'abord être entendues simultanément, puis jouées séparément. 
Perception des battements

Pour stimuler la perception des battements, nous proposons un exercice qui nécessitera une sensibilité plus grande aux interactions qui existent entre les harmoniques : l'accordage des intervalles harmoniques selon les tempéraments juste et tempéré. Bien que cet exercice puisse paraître inhabituel pour certains musiciens, l'accordage selon le tempérament juste exige de prêter attention aux interactions entre les harmoniques, puisqu'il faut tenter de les minimiser. Distinguer ces différences devrait conduire à une plus grande sensibilité pour cette dimension des intervalles harmoniques.

Pour l'intervalle d'unisson et d'octave, les battements sont très faciles à percevoir, puisque la moindre variation de hauteur génèrera des battements sur pratiquement la totalité du spectre des harmoniques. Pour les intervalles de quinte juste, de quarte juste, de tierce, de sixte et de septième mineure, l'étudiant est forcé de prêter attention aux premiers harmoniques communs pour poser son jugement. En effet, les fréquences fondamentales étant trop éloignées pour générer des battements au niveau de la cochlée, les frictions perçues proviennent de l'interaction des premiers harmoniques communs. Pour leur part, les intervalles de secondes, de triton et de septième majeure se prêtent mal à cet exercice d'accordage, puisque les premiers harmoniques communs sont particulièrement éloignés et que le degré de friction est plus élevé. Bien qu'ils ne contraignent pas l'étudiant à percevoir les interactions entre les harmoniques, apprendre à reconnaître la justesse de ces dissonances demeure un objectif pertinent.

\section{Perception de l'harmonicité}

Développer des exercices pour renforcer la perception de l'harmonicité pose certains défis puisque, contrairement aux attributs perceptuels précédents, elle n'est associée à aucun concept musical traditionnel. Il est donc nécessaire d'en créer un qui nous permettra de présenter concrètement ce phénomène et d'attirer ensuite l'attention vers celui-ci dans un contexte pédagogique. Le concept que nous proposons d'introduire est celui de "résolution harmonique ». La résolution harmonique réfère à la recherche d'une ou de plusieurs notes faisant en sorte que les notes de l'intervalle puissent appartenir à une même série harmonique. Afin de bien comprendre comment ce concept pourra nous aider à mieux percevoir les intervalles harmoniques, quelques explications sont requises.

Chaque son musical est accompagné d'une multitude d'harmoniques. L'oreille connaît si bien le schéma habituel des harmoniques qu'elle les fusionne de façon à ne percevoir que la fréquence fondamentale. Or, lorsqu'on écoute deux notes simultanément, l'oreille n'en connaît pas d'emblée le nombre réel. Elle les analyse et tente de les faire appartenir à un même schéma spectral pour leur associer une unique fréquence fondamentale. Sauf dans le cas de l'unisson, les concordances entre les séries harmoniques ne sont jamais parfaites. La plupart du temps, plusieurs résolutions harmoniques sont possibles. Par exemple, la relation d'octave est une relation très fréquente dans la série des harmoniques (on en dénombre huit entre les seize premiers 
harmoniques). Comme l'oreille a l'habitude de percevoir ces différentes relations d'octave entre les harmoniques, l'écoute de l'octave peut théoriquement "réveiller » la sensation de huit notes fondamentales distinctes.

Mais au-delà de l'équivalence théorique de ces résolutions, il faut savoir que les harmoniques naturels ne sont pas d'égales importances. D'abord, l'intensité des harmoniques varie en fonction du timbre des instruments, les harmoniques aigus étant généralement beaucoup moins audibles. Ensuite, l'oreille perçoit les fréquences avec un degré de précision variable. On se souvient que, à partir de $1000 \mathrm{~Hz}$, ou do6, les neurones ne parviennent plus à synchroniser complètement leurs impulsions électriques avec la fréquence de la note entendue. Les harmoniques se situant au-delà de $1000 \mathrm{~Hz}$ sont donc plus difficiles à percevoir. Par ailleurs, la distance entre les harmoniques se resserre dans les harmoniques supérieurs, ce qui complique le travail de la cochlée. Ce problème est naturellement amplifié lorsque les notes jouées à l'instrument se situent dans des registres plus aigus et qu'une portion importante des harmoniques naturels se situe en dehors de la zone de confort de la cochlée. Enfin, plus les résolutions harmoniques sont complexes, plus les fréquences fondamentales communes sont distancées des notes composant l'intervalle. De nombreuses résolutions harmoniques se situent donc à l'extérieur de la plage de fréquences audibles. Ces diverses limites perceptuelles contribuent à réduire le nombre de résolutions harmoniques réellement convenables. En pratique, les résolutions harmoniques les plus stables seront également les plus rapprochées de l'intervalle harmonique.

Dans la figure 2, nous comparons les cinq premières résolutions possibles de l'intervalle d'octave. Bien qu'elles constituent des possibilités théoriques parfaitement convenables, on remarquera rapidement à l'audition que les deux premières résolutions harmoniques, sur les notes $d o$, sont les plus stables. Les troisième et cinquième résolutions, sur les notes fa et la bémol, introduisent une complexité plus grande que le stimulus initial.

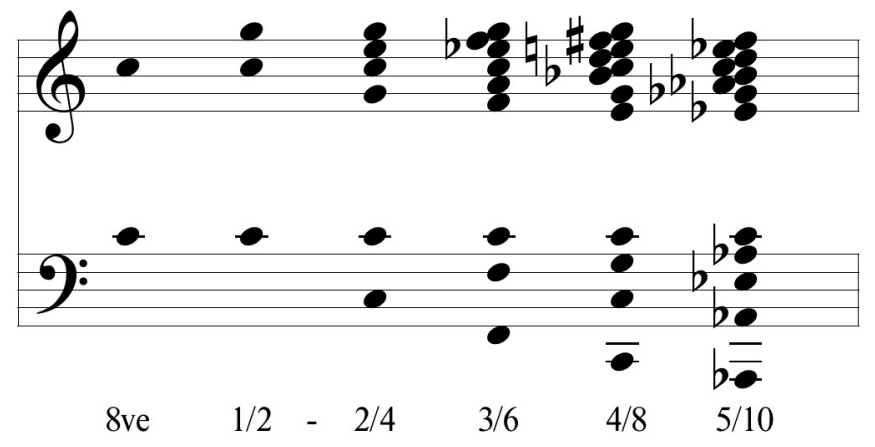

Figure 2 : Résolutions harmoniques de l'octave.

Pour amener l'étudiant à prendre conscience de l'existence de la résolution harmonique des intervalles, le concept de résolution harmonique doit d'abord lui être expliqué et illustré à l'aide d'exemples sonores pertinents. L'étudiant doit ensuite avoir l'occasion d'explorer plusieurs résolutions possibles pour chacun des intervalles et de les classer en fonction de leur degré de concordance. Il peut également s'exercer à reconnaître si, oui ou non, une résolution donnée constitue un choix convenable. Enfin, lorsque la résolution harmonique est bien comprise, elle peut être employée 
pour distinguer des intervalles présentant des similitudes perceptuelles ${ }^{3}$. Par exemple, la quinte et la quarte, qui présentent des similitudes sur le plan des interactions entre les harmoniques, possèdent des résolutions harmoniques complètement différentes (voir exemple audio 7). En effet, l'examen des résolutions nous montre que la quinte se résout sur la note inférieure alors que la quarte se résout sur la note supérieure.

L'exemple audio 7 compare les premières résolutions harmoniques de la quinte et de la quarte.

Exemple audio 7: Comparaison des résolutions harmoniques. Écouter.

APPROCHE INTÉGRÉE POUR LA SALLE DE CLASSE

Le lecteur se demandera certainement comment les différents exercices que nous venons de proposer pourraient être intégrés en classe dans une leçon. Voici une proposition d'approche intégrant tous les éléments que nous avons exposés. Pour chaque séance d'entraînement, nous suggérons de cibler un seul nouvel intervalle harmonique et de réaliser les cinq étapes qui suivent.

Réveiller les oreilles à l'aide d'un exercice de discrimination fine

Pour stimuler la perception de la fréquence fondamentale, commencer par réaliser un exercice de discrimination fine en ciblant quelques notes qui devront être accordées sur différents instruments de musique. Il est également possible d'employer des échantillons sonores préenregistrés et de demander aux étudiants de poser un jugement sur leur justesse. Pour faciliter le travail de sensibilisation, il est également possible de faire entendre une séquence de notes alternant entre la note de référence et d'autres dont les différences de hauteur deviendront graduellement plus subtiles.

L'exemple audio 8 présente une séquence de notes qui varient entre la note de référence $(440 \mathrm{~Hz})$ et d'autres légèrement plus aiguës. Les différences deviennent graduellement plus subtiles : juste, +20 cents, juste, +15 cents, juste, +10 cents, juste, +5 cents, juste, +2 cents, juste, +1 cent, juste.

Exemple audio 8: Discriminer les fréquences rapprochées. Écouter.

\section{Distinguer deux notes entendues simultanément}

Poursuivre ensuite avec un exercice qui encouragera la perception distincte de deux notes jouées simultanément. À cette fin, employer un seul intervalle qui sera joué dans différents registres et sur une variété d'instruments de musique. La tâche de l'étudiant consistera à identifier les notes entendues en s'aidant du clavier et à noter précisément leur hauteur sur une partition (la bonne octave). Lorsque la discrimination est plus difficile, faire entendre une séquence de notes qui alternera entre la présentation simultanée et séparée.

3 Les résolutions harmoniques de tous les intervalles peuvent être consultées en annexe, dans le tableau 1. 
Afin de faciliter la perception distincte de notes jouées simultanément, faites-les entendre d'abord simultanément, puis séparément et, enfin, simultanément. Pour écouter cette procédure, consultez l'exemple audio 9.

Exemple audio 9 : Distinguer deux notes entendues simultanément. Écouter.

Accorder les intervalles harmoniques

La prochaine tâche vise à attirer l'attention de l'étudiant autour des battements caractéristiques de l'intervalle harmonique à l'étude. Elle consiste à poser un jugement sur le degré de justesse d'un intervalle harmonique donné. À cette fin, présenter un intervalle dans sa forme tempérée, puis faire entendre le même intervalle dans sa forme juste en prêtant attention à la différence de battements. Au besoin, faire varier graduellement les hauteurs pour aider à mieux percevoir comment la vitesse des battements est altérée. Pour aider à mieux localiser les battements, il est également possible de jouer la première harmonique commune. Le tableau 3, présenté en annexe, montre les premières harmoniques communes des douze intervalles.

L'exemple audio 10 illustre comment de légères variations de hauteurs permettent de mettre en évidence les interactions entre les harmoniques. À la fin de la séquence, comparez les deux derniers intervalles entendus. Le dernier génère davantage d'interactions que le précédent, alors qu'il est parfaitement tempéré.

Exemple audio 10: Accorder les intervalles harmoniques. Écouter.

Explorer les résolutions harmoniques

$\mathrm{Au}$ clavier, faire expérimenter les différentes résolutions harmoniques possibles pour un intervalle donné, puis comparer leur degré de stabilité/complexité. Après la phase d'expérimentation, déterminer un point de référence pour mémoriser la résolution de l'intervalle à l'étude (exemple : l'unisson, l'octave, la quinte juste, la tierce majeure et la septième mineure se résolvent sur la note inférieure). Le tableau 2 , en annexe, présente une classification pratique des intervalles par type de résolutions harmoniques.

L'exemple audio 11 compare les trois premières résolutions harmoniques de l'intervalle de quinte juste.

Exemple audio 11 : Explorer les résolutions harmoniques. Écouter.

\section{Intégration des connaissances}

En prêtant attention à ses caractéristiques sensorielles, comparer le nouvel intervalle avec les autres étudiés. Quelles sont leurs ressemblances ? En quoi se distinguent-ils les uns des autres ? Remplir une fiche pour consigner leurs ressemblances et leurs différences perceptuelles. Les tableaux 4 et 5 , présentés en annexe, peuvent aider à réaliser cette tâche. En terminant, réaliser une dictée musicale ou un repiquage qui exploitent esthétiquement les qualités particulières de l'intervalle harmonique à l'étude. 
CONCLUSION

Devant les difficultés qu'éprouvent plusieurs étudiants avec la reconnaissance des intervalles harmoniques, plusieurs approches pédagogiques ont été préconisées, mais aucune n'avait encore proposé de moyens concrets pour relever les défis perceptuels liés à leur étude. Avec une meilleure compréhension des mécanismes de perception auditive et d'apprentissage perceptuel, nous avons proposé des exercices qui visent à renforcer la perception sonore : discrimination fine des fréquences, perception distincte de notes jouées simultanément, interactions entre les harmoniques et harmonicité.

Bien qu'elles n'aient pas encore été expérimentées en classe, nos propositions reposent sur des fondations théoriques solides et nous avons bon espoir que les différents exercices présentés constitueront des moyens efficaces pour : 1) créer un contexte favorable à l'apprentissage perceptuel ; 2) attirer l'attention de l'étudiant autour des différents indices sonores qui influencent sa perception des intervalles harmoniques ; 3) renforcer, sur le plan neurologique, la perception de ces différents attributs sonores ; 4) favoriser un apprentissage plus profond et durable des intervalles harmoniques, notamment en offrant à l'étudiant davantage d'outils pour décrire les caractéristiques des différents intervalles étudiés.

Notre " approche intégrée pour la salle de classe " méritera d'être testée dans le cadre d'une recherche expérimentale pour en mesurer d'abord les effets sur le plan de l'apprentissage perceptuel et pour comparer ensuite son efficacité pédagogique avec d'autres approches, notamment celle de Ponsati et al. (2016).

Dans l'immédiat, l'approche intégrée et les différents exercices peuvent être employés dans la classe de formation auditive pour enrichir les curriculums habituels ou pour répondre à des besoins pédagogiques ponctuels. Malgré l'absence de matériel adapté actuellement accessible, tous les exercices proposés peuvent être réalisés en classe à l'aide des instruments de musique que possèdent les étudiants.

L'étude des intervalles harmoniques nous offre une belle occasion pour développer l'oreille musicale. Loin d'alourdir les curriculums d'études actuels, nous espérons que notre approche, axée sur l'entraînement perceptuel, facilitera l'étude des intervalles harmoniques et favorisera un apprentissage plus durable.

Note

Cet article a été rédigé dans le cadre de l'examen rétrospectif prévu au programme de doctorat en éducation musicale de l'Université Laval. L'auteur a reçu l'appui financier de la bourse d'études supérieures du Canada Joseph-Armand-Bombardier $\mathrm{du}$ Conseil de recherche en sciences humaines du Canada (CRSH). 


\section{BIBLIOGRAPHIE}

Balzano, Gerald, J., et Barry W. Liesch (1982), «The Role of Chroma and Scalestep in the Recognition of Musical Intervals In and Out of Context ", Psychomusicology. A Journal of Research in Music Cognition, vol. 2, no 2, p. 3-31.

Bao, Min., et al. (2010), «Perceptual Learning Increases the Strength of the Earliest Signals in Visual Cortex », The Journal of Neuroscience, vol. 30, n 45, p. 15080-15084.

Bendor, Daniel, et Xiaoqin Wang (2005), « The Neuronal Representation of Pitch in Primate Auditory Cortex », Nature, vol. 436, n 7054 , p. 1161-1165.

Bernstein, Joshua G. W., et Andrew J. Oxenham (2008), « Harmonic Segregation Through Mistuning Can Improve Fundamental Frequency Discrimination ", The Journal of the Acoustical Society of America, vol. 124, $\mathrm{n}^{\circ}$ 3, p. 1653-1667.

Bidelman, Gavin M., et Ananthanarayan Krishnan (2009), " Neural Correlates of Consonance, Dissonance, and the Hierarchy of Musical Pitch in the Human Brainstem », The Journal of Neuroscience, vol. 29, no 42, p. 13165-13171.

Bowling, Daniel L., et Dale Purves (2015), " A Biological Rationale for Musical Consonance », Proceedings of the National Academy of Sciences, vol. 112, $\mathrm{n}^{\circ}$ 36, p. 11155-11160.

Bosnyak, Daniel J., Eaton, Robert A., et Larry E. Roberts (2004), « Distributed Auditory Cortical Representations Are Modified When Non-musicians Are Trained at Pitch Discrimination with $40 \mathrm{~Hz}$ Amplitude Modulated Tones », Cerebral Cortex, vol. 14, nº 10, p. 1088-1099.

Carcagno, Samuelle, et Christopher J. Plack (2011), " Subcortical Plasticity Following Perceptual Learning in a Pitch Discrimination Task ", Journal of the Association for Research in Otolaryngology, vol. 12 , n 1 , p. 89-100.

Chouard, Claude-Henri (2001), L'oreille musicienne. Les chemins de la musique de l'oreille au cerveau, Paris, Gallimard.

Costa, Marco, Bitti, Pio E. R., et Luisa Bonfiglioli (2000), « Psychological Connotations of Harmonic Musical Intervals », Psychology of Music, vol. 28, n 1, p. 4-22.

Cousineau, Marion, McDermott, Josh H., et Isabelle Peretz (2012), «The Basis of Musical Consonance as Revealed by Congenital Amusia », Proceedings of the National Academy of Sciences, vol. 109, n 48, p. 19858-19863.

Demany, Laurent, et Christophe Ramos (2005), « On the Binding of Successive Sounds. Perceiving Shifts in Nonperceived Pitches ", Journal of the Acoustical Society of America, vol. 117, n 2, p. 833841.

Demany, Laurent, et Catherine Semal (2002), « Learning to Perceive Pitch Differences », The Journal of the Acoustical Society of America, vol. 111, no 3, p. 1377-1388.

Deutch, Diana (2013), "Grouping Mechanisms in Music », dans Diana Deutch (dir.), The Psychology of Music, $3^{\mathrm{e}}$ édition, London, Elsevier, p. 183-248.

Furmanski, Christopher S., Schluppeck, Denis, et Stephen A. Engel (2004), « Learning Strengthens the Response of Primary Visual Cortex to Simple Patterns », Current Biology, vol. 14, n 7, p. 573578.

Garner, Wendell R. (1976), «Interaction of Stimulus Dimensions in Concept and Choice Processes », Cognitive Psychology, vol. 8, no 1, p. 98-123.

Goldstein, E. Bruce (2010), Sensation and Perception, $8^{\mathrm{e}}$ édition, Belmont, Wadsworth Cengage Learning.

Golstone, Robert L. (1994), «Influences of Categorization on Perceptual Discrimination », Journal of Experimental Psychology, vol. 123, $\mathrm{n}^{\circ}$ 2, p. 178-200.

Goldstone, Robert L., et Lisa Byrge (2015), " Perceptual Learning », dans Mohen Matthen (dir.), The Oxford Handbook of Philosophy of Perception, Oxford, Oxford University Press. 
Goldstone, Robert L., et Andrew T. Hendrickson (2010), " Categorical Perception », Wiley Interdisciplinary Reviews. Cognitive Science, vol. 1, n 1, p. 69-78.

Goldstone, Robert L., et Mark Steyvers (2001), «The Sensitization and Differentiation of Dimensions During Category Learning ", Journal of Experimental Psychology. General, vol. 130, n 1, p. 116139.

Huron, David (1989), "Voice Denumerability in Polyphonic Music of Homogeneous Timbres ", Music Perception, vol. 6, nº 4, p. 361-382.

Kameoka, Akio, et Mamoru Kuriyagawa (1969), "Consonance Theory Part II. Consonance of Complex Tones and its Calculation Method ", Journal of the Acoustical Society of America, vol. 45, p. $1460-1469$.

Karpinski, Gary S. (2000), Aural Skills Acquisition. The Development of Listening, Reading, and Performing Skills in College-level Musicians, New York, Oxford University Press.

Keislar, Douglas F. (1989), «Psychoacoustic Factors in Musical Intonation. Beats, Interval Tuning, and Inharmonicity ", Thèse de doctorat, Stanford University.

Killam, Rosemary N., Lorton Jr., Paul V., et Earl D. Schubert (1975), « Interval Recognition. Identification of Harmonic and Melodic Intervals ", Journal of Music Theory, vol. 19, n 2, p. 212234.

Langner, Gerald, et Michael Ochse (2006), « The Neural Basis of Pitch and Harmony in the Auditory System », Musicae Scientiae, vol. 10, n 1, p. 185-208.

Lee, Kyung M. (2011), « Neural Representation of Musical Intervals in the Human Brainstem », Thèse de doctorat, Northwestern University.

Lee, Kyung M., et al. (2009), "Selective Subcortical Enhancement of Musical Intervals in Musicians », The Journal of Neuroscience, vol. 29, $\mathrm{n}^{\circ} 18$, p. 5832-5840.

Loh, Christian S. (2007), "Choice and Effects of Instrument Sound in Aural Training », Music Education Research, vol. 9, n 1, p. 129-143.

Maher, Timothy F. (1976), " "Need for Resolution". Ratings for Harmonic Musical Intervals. A Comparison Between Indians and Canadians ", Journal of Cross-Cultural Psychology, vol. 7, $\mathrm{n}^{\circ} 3$, p. 259-276.

Maher, Timothy F. (1980), «A Rigorous Test of the Proposition That Musical Intervals Have Different Psychological Effects », The American Journal of Psychology, vol. 93, n 2, p. 309-327.

McDermott, Josh H., Lehr, Andriana J., et Andrew J. Oxenham (2010), «Individual Differences Reveal the Basis of Consonance ", Current Biology, vol. 20, n 11, p. 1035-1041.

McDermott, Josh. H., et Andrew J. Oxenham (2008), « Music Perception, Pitch, and the Auditory System ", Current Opinion in Neurobiology, vol. 18, no 4, p. 452-463.

McLachlan, Neil, et al. (2013), "Consonance and Pitch ", Journal of Experimental Psychology. General, vol. 142, n 4, p. 1142-1158.

Micheyl, Christophe, et Andrew J. Oxenham (2010), " Pitch, Harmonicity and Concurrent Sound Segregation. Psychoacoustical and Neurophysiological Findings ", Hearing Research, vol. 266, n 1 , p. $36-51$.

Miyazono, Hiromitsu, Glasberg, Brian R., et Brian C. J. Moore (2010), « Perceptual Learning of Fundamental Frequency Discrimination. Effects of Fundamental Frequency, Harmonic Number, and Component Phase ", Journal of the Acoustical Society of America, vol. 128, n 6, p. 3649-3657.

Musacchia, Gabriella, et al. (2007), «Musicians Have Enhanced Subcortical Auditory and Audiovisual Processing of Speech and Music », Proceedings of the National Academy of Sciences, vol. 104, n 40, p. $15894-15898$.

Needham, Amy, Dueker, Gwenden, et Gregory Lockhead (2005), «Infants' Formation and Use of Categories to Segregate Objects ", Cognition, vol. 94, n 3, p. 215-240.

Oelmann, Hella, et Bruno Laeng (2009), "The Emotional Meaning of Harmonic Intervals », Cognitive Processing, vol. 10, n 2, p. 113-131. 
Oxenham, Andrew J. (2013), "The Perception of Musical Tones », dans Diana Deutch (dir.), The Psychology of Music, $3^{e}$ édition, London, Elsevier, p. 1-33.

Pantev, Christo, et al. (1998), "Increased Auditory Cortical Representation in Musicians ", Nature, vol. $392, \mathrm{n}^{\circ} 6678$, p. 811-814.

Plomp, Reinier, et Willem J. M. Levelt (1965), "Tonal Consonances and Critical Bandwidth », Journal of the Acoustical Society of America, vol. 38, n 4, p. 548-560.

Ponsati, Imma, et al. (2016), "Students' Performance When Aurally Identifying Musical Harmonic Intervals. Experimentation of a Teaching Innovation Proposal », International Journal of Music Education, vol. 34, no 4, p. 445-458.

Quinn, Paul C., et Ramesh R. Bhatt (2006), «Are Some Gestalt Principles Deployed More Readily Than Others During Early Development? The Case of Lightness Versus Form Similarity ", Journal of Experimental Psychology. Human Perception and Performance, vol. 32, n 5, p. 1221-1230.

Roederer, Juan G. (2008), The Physics and Psychophysics of Music. An Introduction, $4^{e}$ édition, New York, Springer.

Rogers, Michael (2004), Teaching Approaches in Music Theory. An Overview of Pedagogical Philosophies, Carbondale, Southern Illinois University Press.

Samplaski, Art (2005), "Interval and Interval class Similarity. Results of a Confusion Study », Psychomusicology. A Journal of Research in Music Cognition, vol. 19, $\mathrm{n}^{\circ}$ 1, p. 59-74.

Schellenberg, E. Glenn, et Laurel J. Trainor (1996), "Sensory Consonance and the Perceptual Similarity of Complex-tone Harmonic Intervals. Tests of Adult and Infant Listeners ", Journal of the Acoustical Society of America, vol. 100, n ${ }^{\circ}$ 5, p. 3321-3328.

Schön, Daniele, et al. (2005), "Sensory Consonance. An ERP Study », Music Perception, vol. 23, n 2, p. 105-117.

Schneider, Peter, et al. (2002), "Morphology of Heschl's Gyrus Reflects Enhanced Activation in the Auditory Cortex of Musicians ", Nature Neuroscience, vol. 5, n 7, p. 688-694.

Smith, J. David, et al. (1994), "What Child is This? What Interval Was That? Familiar Tunes and Music Perception in Nnovice Listeners ", Cognition, vol. 52, $\mathrm{n}^{\circ}$ 1, p. 23-54.

Sowden, Paul T., Davies, Ian R. L., et Penny Roling (2000), «Perceptual Learning of the Detection of Features in X-ray Images. A Functional Role for Improvements in Adults' Visual Sensitivity? », Journal of Experimental Psychology. Human Perception and Performance, vol. 26, n ${ }^{\circ}$ 1, p. 379-390.

Stainsby, Thomas, et Ian Cross (2009), "The Perception of Pitch ", dans Susan Hallam, Ian Cross et Michael Thaut (dir.), The Oxford Handbook of Music Psychology, Oxford, Oxford University Press, p. 47-58.

Stolzenburg, Frieder (2015), « Harmony Perception by Periodicity Detection », Journal of Mathematics and Music, vol. 9, $\mathrm{n}^{\circ}$ 3, p. 215-238.

Tanaka, James W., et Tim Curran (2001), «A Neural Basis for Expert Object Recognition», Psychological Science, vol. $12, \mathrm{n}^{\circ} 1$, p. 43-47.

Thompson, William F. (2013), "Intervals and Scales », dans Diana Deutch (dir.), The Psychology of Music, $3^{\mathrm{e}}$ édition, London, Elsevier, p. 107-140.

Tramo, Mark J., et al. (2001), « Neurobiological Foundations For the Theory of Harmony in Western Tonal Music », Annals of the New York Academy of Sciences, vol. 930, n 1, p. 92-116.

Trainor, Laurel J., et Erin E. Hannon (2013), " Musical Development », dans Diana Deutch (dir.), The Psychology of Music, $3^{\mathrm{e}}$ édition, London, Elsevier, p. 423-497.

Watanabe, Takeo, Nanez, José E., et Yuka Sasaki (2011), « Perceptual Learning Without Perception », Nature, vol. 413, p. 844-848.

Weinberger, Norman M. (1993), "Learning-induced Changes of Auditory Receptive Fields ", Current Opinion in Neurobiology, vol. 3, n 4, p. 570-577.

Willems, Edgar, et Jacques Chapuis (1996), Canciones de intervalos y acordes, Barcelone, Editions Pro 
Musica.

Wuthrich, Carol E., et Thomas Tunks (1989), «The Influence of Presentation Time Asynchrony on Music Interval Perception », Psychomusicology. A Journal of Research in Music Cognition, vol. 8, $\mathrm{n}^{\circ} 1$, p. 31-46.

Zarate, Jean M., Ritson, Caroline R., et David Poeppel (2012), "Pitch-interval Discrimination and Musical Expertise. Is the Semitone a Perceptual Boundary? », Journal of Acoustical Society of America, vol. 132, no 2, p. 984-993.

Zatorre, Robert J. (1983), « Category-boundary Effects and Speeded Sorting with a Harmonic Musicalinterval Continuum. Evidence for Dual Processing ", Journal of Experimental Psychology. Human Perception \& Performance, vol. 9, n ${ }^{\circ}$ 5, p. 739-752.

ANNEXe A : Résolutions harmoniques.

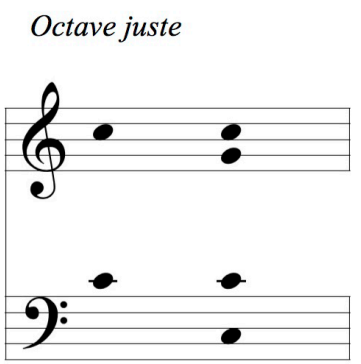

\section{Quinte juste}

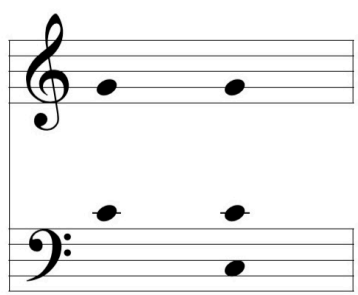

Sixte majeure

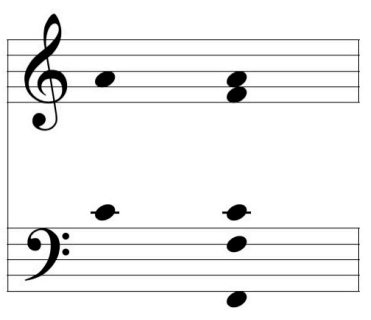

Septième mineure

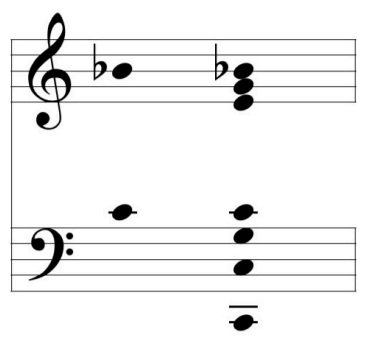


Sixte mineure

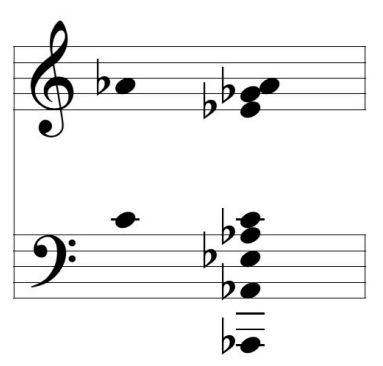

Triton

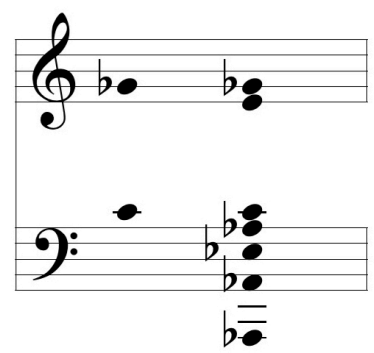

Septième majeure

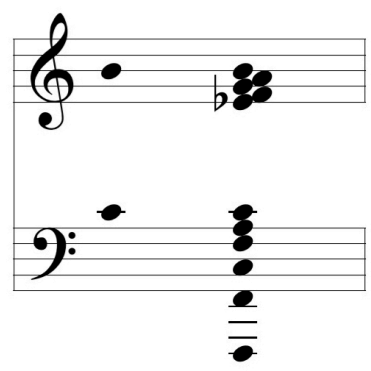

Tierce mineure

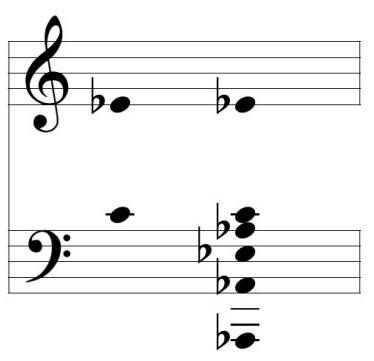

Seconde majeure

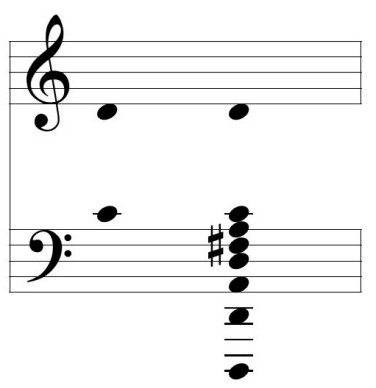

Seconde mineure

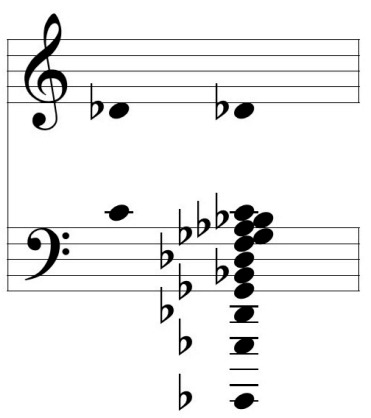




\section{ANNEXE B : Classification pratique des résolutions harmoniques.}

\begin{tabular}{|c|c|}
\hline \multirow{4}{*}{ Note inférieure de l'intervalle } & Octave juste \\
\hline & Quinte juste \\
\hline & Tierce majeure \\
\hline & Septième mineure \\
\hline \multirow{3}{*}{ Note supérieure de l'intervalle } & Quarte juste \\
\hline & Sixte mineure \\
\hline & Seconde majeure \\
\hline \multirow{2}{*}{$\begin{array}{l}\text { Autre note correspondant à la } \\
\text { fondamentale d'un accord majeur }\end{array}$} & Sixte majeure \\
\hline & Tierce mineure \\
\hline \multirow{3}{*}{ Autre note éloignée } & Triton \\
\hline & Septième majeure \\
\hline & Seconde mineure \\
\hline
\end{tabular}


ANNEXE C : Premiers harmoniques communs (perception des battements).

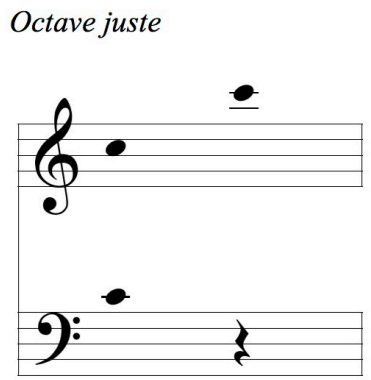

Quinte juste

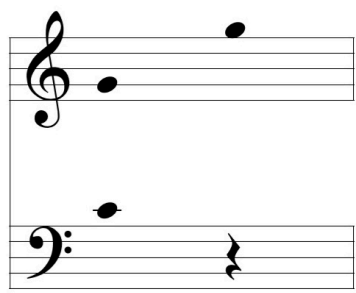

Quarte juste

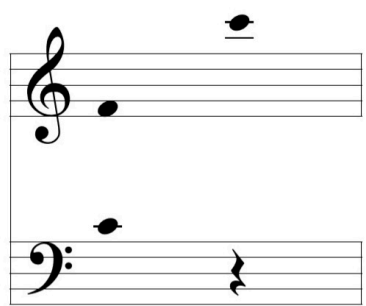

Tierce majeure

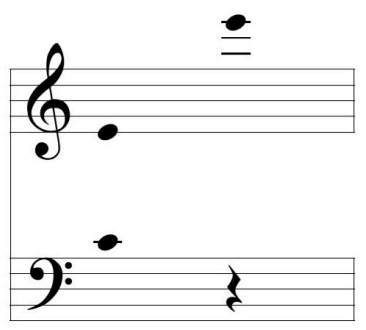

Sixte majeure

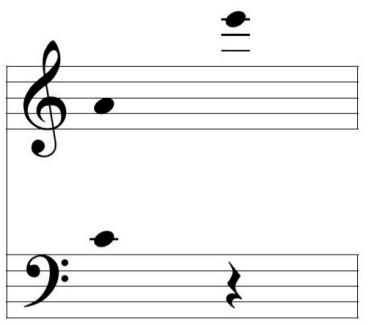

Septième mineure

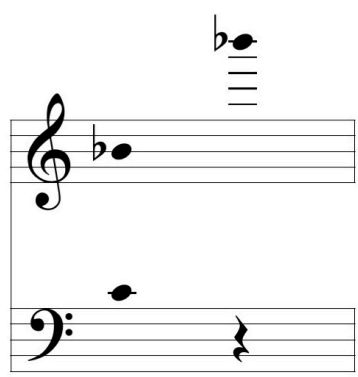



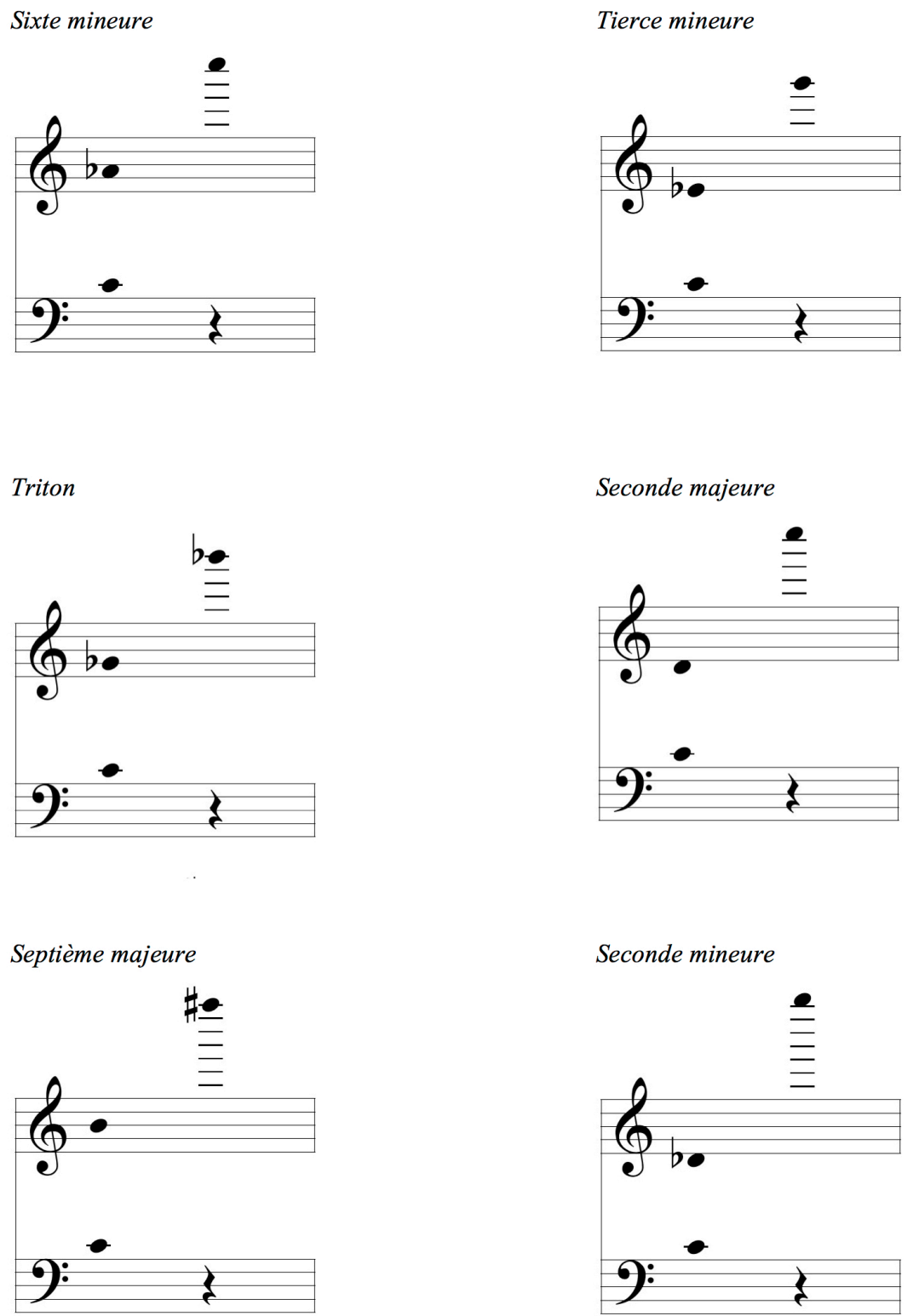


\section{ANNEXE D : Classification pratique des harmoniques communs.}

\begin{tabular}{|c|c|}
\hline \multirow{4}{*}{ Doublure de la note inférieure } & Octave juste \\
\hline & Quarte juste \\
\hline & Sixte mineure \\
\hline & Seconde majeure \\
\hline \multirow{3}{*}{ Doublure de la note supérieure } & Quinte juste \\
\hline & Tierce majeure \\
\hline & Septième mineure \\
\hline \multirow{2}{*}{$\begin{array}{l}\text { Autre note correspondant à la quinte } \\
\text { d'un accord mineur }\end{array}$} & Sixte majeure \\
\hline & Tierce mineure \\
\hline \multirow{3}{*}{ Autre note éloignée } & Triton \\
\hline & Septième majeure \\
\hline & Seconde mineure \\
\hline
\end{tabular}

\section{ANNEXE E : Comparaison des intervalles harmoniques.}

\begin{tabular}{|l|l|l|}
\hline & Harmoniques communes & Résolution harmonique \\
\hline Seconde mineure & Note éloignée & Résolution éloignée \\
\hline Seconde majeure & Note inférieure & Note supérieure \\
\hline Tierce mineure & Autre note (accord mineur) & Autre note (accord majeur) \\
\hline Tierce majeure & Note supérieure & Note inférieure \\
\hline Quarte juste & Note inférieure & Note supérieure \\
\hline Triton & Tierce note éloignée & Note éloignée \\
\hline Quinte juste & Note supérieure & Note inférieure \\
\hline Sixte mineure & Note inférieure & Autre note (accord majeur) \\
\hline Sixte majeure & Autre note (accord mineur) & Note supérieure \\
\hline Septième mineure & Note supérieure & Note inférieure \\
\hline Septième majeure & Note éloignée & Note éloignée \\
\hline Octave & Notes inférieure et supérieure & Notes inférieure et supérieure \\
\hline
\end{tabular}

